\title{
Towards the Mechanism of Oxygen Vacancy Formation \& Ordering via Tracking of Beam-Induced Dynamics and Density Functional Theory
}

\author{
Axiel Yaël Birenbaum ${ }^{1}$, Liang Qiao², Valentino R. Cooper ${ }^{1}$ and Albina Borisevich ${ }^{1}$ \\ 1. Material Science \& Technology Division, Oak Ridge National Laboratory, Oak Ridge, TN. \\ 2. School of Materials, University of Manchester, Oxford Rd, Manchester, UK.
}

Energy applications such as oxide-fuel solid cells (SOFC) require fast oxygen transport. The formation and subsequent transport of oxygen vacancies and ions depend subtly on the physical and electrochemical properties of the oxide electrolyte. A good candidate for this is the lanthanum cobaltite perovskite series, due to their excellent oxygen transport properties [1]. The system often exhibits oxygen off-stoichiometry, and several vacancy-ordered phases have been reported, with vacancies segregating into planes that show up as "stripes" in STEM images. These "striped" phases have been observed to form either parallel or perpendicular to the substrate (hence coupled to strain) when $\mathrm{LaCoO}_{3-\delta}$ films are grown on $\left(\begin{array}{lll}0 & 0 & 1\end{array}\right)$ $\left(\mathrm{LaAlO}_{3}\right)_{0.3}\left(\mathrm{Sr}_{2} \mathrm{AlTaO}_{6}\right)_{0.7}$ [1] and $\left(\begin{array}{lll}0 & 0 & 1\end{array}\right) \mathrm{SrTiO}_{3}$ [2], respectively; a multidirectional pattern was observed in $\mathrm{La}_{0.5} \mathrm{Sr}_{0.5} \mathrm{CoO}_{3-\delta}$ grown on $\left(\begin{array}{lll}1 & 1 & 0\end{array}\right) \mathrm{SrTiO}_{3}$ [3]. An interesting question is if oxygen vacancies order in $\mathrm{LaCoO}_{3-\delta}$, when grown on $\mathrm{SrTiO}_{3}\left(\begin{array}{lll}1 & 1 & 1\end{array}\right)$, where all "stripe" directions are strongly coupled to strain.

It has recently been established that vacancy-ordered dark "stripes", are accompanied by the local expansion of the lattice that is proportional to the change in the local oxygen content. [2]. Given the substantial expansion involved (up to 20\%), it is conceivable that the epitaxial strain can be used to tailor local oxygen content and vacancy ordering. While lattice-expansion based approach has proven successful for monitoring dynamic changes in oxygen content during electron beam-induced ordering [5], the energetics involved in forming and ordering vacancies remain unknown. Even after the initial and final states are understood, the energetics of intermediate stages of the process are not yet clarified, and can impact the oxygen transport properties.

Employing a combination of density functional theory (DFT) and scanning transmission electron microscopy (STEM) measurements we examine the energetics and dynamics of oxygen vacancy ordering in the $\left(\begin{array}{lll}1 & 1 & 1\end{array}\right)$ oriented $\mathrm{LaCoO}_{3-\delta}$ on $\mathrm{SrTiO}_{3}$. We used $\mathrm{NION}{ }^{\circledR}$ UltraSTEM operated at $200 \mathrm{kV}$. The films studied were oxygen deficient as-grown. The DFT calculations were performed on a $\sqrt{ } 2 \times \sqrt{ } 2 \times 6$ perovskite unit cells in order to evaluate the rhombohedral perovskite phase $\mathrm{LaCoO}_{3}$ in a unit cell which is commensurate with the oxygen deficient phase $\mathrm{LaCoO}_{3-1 / 3}$. We used PAW pseudopotentials and PBEsol+U functionals, where $\mathrm{U}_{\mathrm{La}}=6.0 \mathrm{eV}$ and $\mathrm{U}_{\mathrm{Co}}=4.0 \mathrm{eV}$, and partially ignored magnetism.

Here, we lay the groundwork for understanding the dynamics of oxygen vacancy formation and ordering in the $\mathrm{LaCoO}_{3-\delta}$ series. First, we demonstrate that beam-induced oxygen vacancy ordering in $\mathrm{LaCoO}_{3-\delta}$ grown on $\mathrm{SrTiO}_{3}\left(\begin{array}{lll}1 & 1 & 1\end{array}\right)$ can be observed on a timescale of $\sim 15$ minutes. We examine the oxygen redistribution by tracking the La-La distance along $\left(\begin{array}{lll}1 & 0 & 0\end{array}\right)$ as a function of time, see Figure 1 . In addition to tracking local concentration, by comparing the average La-La distance at the initial and final stage, we can calculate the global averages as a function of time, allowing us to distinguish between redistribution of already present vacancies and beam-induced vacancy formation. Finally, using DFT we are able to perform an analysis of the step-by-step sequence of vacancy formation and ordering, under two different strain states. We not only

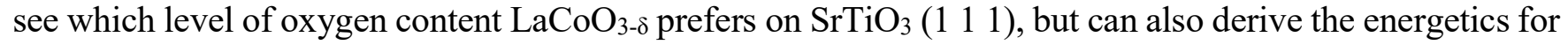
the vacancy formation and ordering process. We conclude by expanding our static analysis of the ordering 
transition to transport anisotropy [6].

References:

[1] V. S. Bagotsky in "Fuel cells: problems and solutions", ed. ECS, (Wiley, Hoboken NJ).

[2] Y. M. Kim et al, Nature Materials 11 (2012), p. 888.

[3] N. Biškup et al, Physical Review Letters 112 (2014).

[4] J. Gazquez et al, Applied Physics Letters Materials 1 (2013).

[5] J. H. Jang et al, ACS Nano 11 (2017), p. 6942.

[6] We acknowledge the contribution of the late Dr. Michael D. Biegalski for growing the sample.

Research was sponsored by the US Department of Energy, Office of Science, Basic Energy Sciences,

Materials Sciences and Engineering Division. This research used resources of the National Energy

Research Scientific Computing Center, which was supported by the Office of Science of the US

Department of Energy under Contract No. DE-AC02-05CH11231.

a)

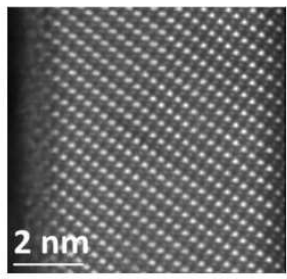

b)

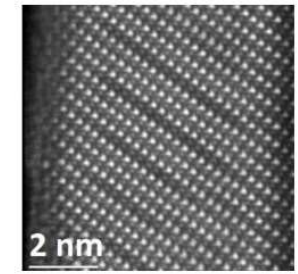

c)

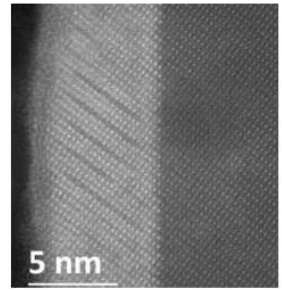

d)
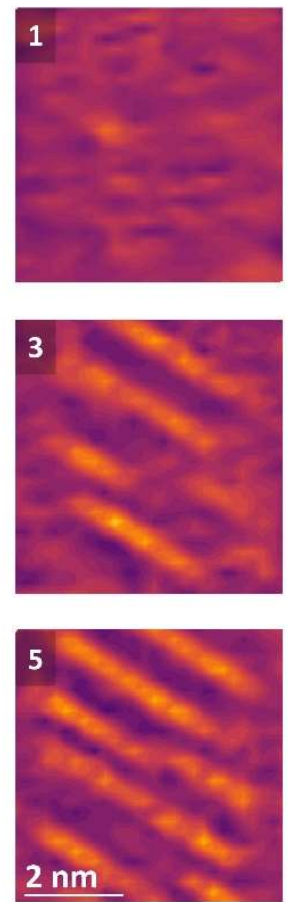
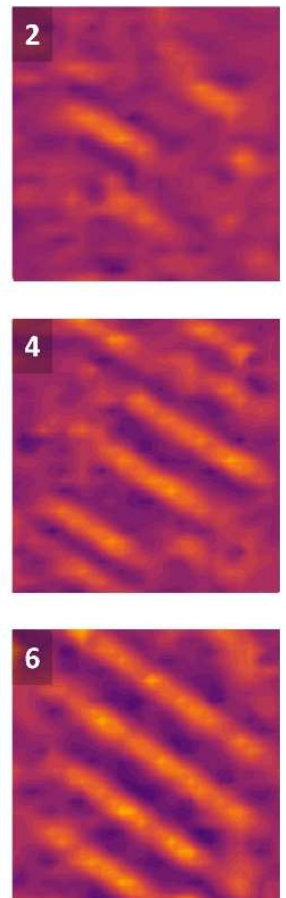

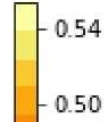

0.46

$-0.42$

$-0.38$

$-0.34$

0.30

$\mathrm{nm}$

Figure 1. Evolution of $\mathrm{LaCoO}_{3-\delta}$ thin films over 15 min of electron irradiation. (a-c) Single HAADF STEM images taken (a) at the start and (b, c) at the end of the procedure. (d) A selection of La-La (1 00 ) distance maps computed from frames in 7x200 image sequences taken between (a) and (b, c); local spacing is given by color scale (in nm). Images 1-6 correspond to following frames in order: 1-003, 1-184, 2-141, 4-022, 5037, 7-197.

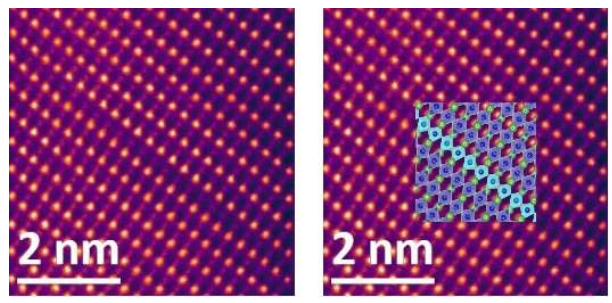

Figure 2. HAADF images of a single "stripe" in $\mathrm{LaCoO}_{3-\delta}$. Both images are identical, the right one having the DFT model superposed. Green atoms are La, blue are Co, the dark polygons representing oxygen octahedra and the light blue oxygen tetrahedra. 